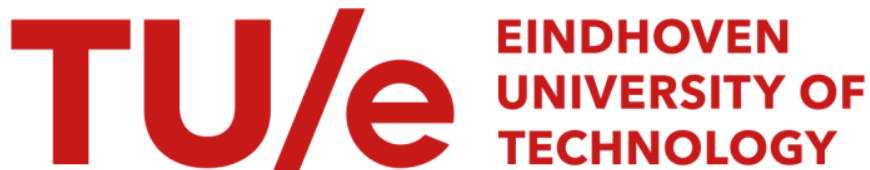

\section{Spectral narrowing in coherent Rayleigh-Brillouin scattering}

Citation for published version (APA):

Manteghi, A., Dam, N. J., Meijer, A. S., Wijn, de, A. S., \& Water, van de, W. (2011). Spectral narrowing in coherent Rayleigh-Brillouin scattering. Physical Review Letters, 107(17), 173903-1/4. [173903].

https://doi.org/10.1103/PhysRevLett.107.173903

DOI:

10.1103/PhysRevLett.107.173903

Document status and date:

Published: 01/01/2011

\section{Document Version:}

Publisher's PDF, also known as Version of Record (includes final page, issue and volume numbers)

\section{Please check the document version of this publication:}

- A submitted manuscript is the version of the article upon submission and before peer-review. There can be important differences between the submitted version and the official published version of record. People interested in the research are advised to contact the author for the final version of the publication, or visit the $\mathrm{DOI}$ to the publisher's website.

- The final author version and the galley proof are versions of the publication after peer review.

- The final published version features the final layout of the paper including the volume, issue and page numbers.

Link to publication

\section{General rights}

Copyright and moral rights for the publications made accessible in the public portal are retained by the authors and/or other copyright owners and it is a condition of accessing publications that users recognise and abide by the legal requirements associated with these rights.

- Users may download and print one copy of any publication from the public portal for the purpose of private study or research.

- You may not further distribute the material or use it for any profit-making activity or commercial gain

- You may freely distribute the URL identifying the publication in the public portal.

If the publication is distributed under the terms of Article 25fa of the Dutch Copyright Act, indicated by the "Taverne" license above, please follow below link for the End User Agreement:

www.tue.nl/taverne

Take down policy

If you believe that this document breaches copyright please contact us at:

openaccess@tue.nl

providing details and we will investigate your claim. 


\title{
Spectral Narrowing in Coherent Rayleigh-Brillouin Scattering
}

\author{
A. Manteghi* and N. J. Dam \\ Mechanical Engineering Department, Eindhoven University of Technology, \\ PO Box 513, 5600 MB Eindhoven, The Netherlands \\ A. S. Meijer and A. S. de Wijn \\ Institute for Molecules and Materials, Radboud University of Nijmegen, \\ PO Box 9010/51, 6500 GL Nijmegen, The Netherlands
}

\author{
W. van de Water \\ Physics Department, Eindhoven University of Technology, PO Box 513, 5600 MB Eindhoven, The Netherlands
} (Received 12 July 2011; published 18 October 2011)

\begin{abstract}
Coherent Rayleigh-Brillouin scattering is a four-wave mixing technique that provides information on various physical properties of the scattering medium in the spectral domain. Being based on density gratings generated by dipole forces, the method requires two pump beams of sufficient spectral width to span the full response bandwidth of the scattering medium. We provide experimental data on the scattered spectrum as a function of the coherence between the two pump beams and derive the corresponding pump beam spectrum. We argue that all experiments on coherent Rayleigh-Brillouin scattering to date, have, in fact, been performed in the incoherent regime and show that orders of magnitude in scattering efficiency are to be expected when the experiments are performed with bandwidth-limited picosecond laser pulses.
\end{abstract}

DOI: 10.1103/PhysRevLett.107.173903

PACS numbers: 42.25.Kb, 42.65.Es

In coherent Rayleigh-Brillouin scattering (CRBS), dipole forces induce density gratings in a gas in the intersection region of two nearly counterpropagating, pulsed pump laser beams (see Fig. 1). The dynamics of this process is probed via the spectrum of a narrow-band probe laser beam that is Bragg-scattered off the induced density gratings. This spectrum provides information on various properties of the scattering medium, like heat conductivity, bulk viscosity, and shear viscosity, if its temperature and density are known, and vice versa. Since the length and time scales of the induced gratings are short, corresponding to sound at optical wavelengths, the properties of the gas are probed in unusual kinetic regimes [1].

In order to record a faithful scattering spectrum, the spectral width of the forcing should encompass the full width of the response of the medium, which is on the order of the Doppler linewidth. For this reason, all experiments to date have been performed using essentially independent pump beams derived from broad-band Nd:YAG lasers with a nanosecond pulse duration [1-4]. In this Letter, we show how the coherence properties of the interfering pump beams affect the dipolar forcing. We present data on the scattered spectrum as a function of the coherence between the two pump beams and show why previous CRBS experiments have necessarily operated in the incoherent regime. Finally, we predict an efficiency gain of 2 orders of magnitude if transform-limited picosecond pulses are used for forcing.

Under conditions of weak excitation, the scattered spectrum is a product of the forcing spectrum and the response of the medium (the Rayleigh-Brillouin effect) $[2,4,5]$. Here, we only deal with the former, the forcing spectrum, which depends on the coherence properties of the pump beams.

In CRBS, density perturbations are induced by dipole forces, $\mathbf{A} \propto \nabla\left(E_{1} E_{2}^{*}\right)$. The perturbation of the positionvelocity distribution function is described by the linearized

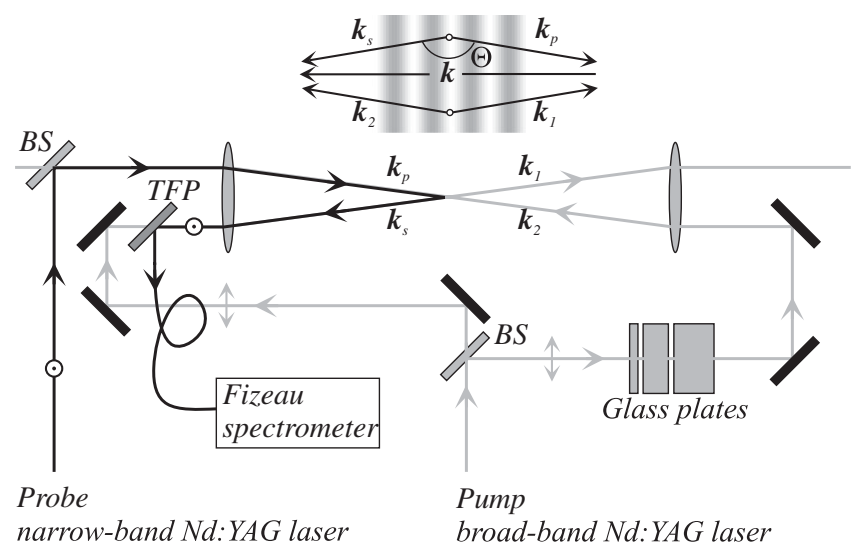

FIG. 1. Schematic diagram of the experimental setup used for CRBS (not to scale). The counterpropagating pump beams with wave vectors $\mathbf{k}_{1}$ and $\mathbf{k}_{2}$ are indicated in gray; the probe laser beam with wave vector $\mathbf{k}_{p}$ and the scattered light beam with $\mathbf{k}_{s}$ are black. The scattered light is collected in a single-mode optical fiber and transported to the Fizeau spectrometer. A thin film polarizer is indicated by TFP, beam splitters by BS. The polarization directions are indicated by $₫$ and $\odot$. The arrangement of the wave vectors corresponding to the phase-matching condition is indicated. $\mathbf{k}=\mathbf{k}_{2}-\mathbf{k}_{1}$ is the scattering wave vector. The glass plates control the time delay between the two pump beams. 
Boltzmann equation, and the density perturbation, and thus the refractive index perturbation $\Delta n$, depends linearly on $A$. The scattered light intensity, $I_{s}(k, \omega) \propto|\Delta n(k, \omega)|^{2}$, then involves a fourth-order moment of the electric fields. The induced gratings are probed on a nanosecond time scale, that is, the scattering signal is integrated over many coherence times of the (broad-band) pump laser. We will approximate this time average by an ensemble average, $\langle\cdots\rangle$. The forcing spectrum of the density gratings can then be written as $\left\langle|A(\omega)|^{2}\right\rangle \propto\left\langle\left|E_{1}(\omega) E_{2}^{*}(\omega)\right|^{2}\right\rangle$. In the time domain, we write the fields as $E(t)=a(t) u(t)$, in which $a(t)$ is the (real, deterministic) field envelope and $u(t)$ contains the (complex, stochastic) time dependence. Assuming that one field is the time-delayed version of the other (delay time $\tau_{d}$, adjustable in the experiment by inserting glass plates; see Fig. 1), we then have

$$
\begin{aligned}
\left\langle|A(\omega)|^{2}\right\rangle \propto & \iint d t d t^{\prime} e^{-l \omega\left(t-t^{\prime}\right)}\left\langle a(t) u(t) a\left(t-\tau_{d}\right)\right. \\
& \left.\times u^{*}\left(t-\tau_{d}\right) a\left(t^{\prime}\right) u^{*}\left(t^{\prime}\right) a\left(t^{\prime}-\tau_{d}\right) u\left(t^{\prime}-\tau_{d}\right)\right\rangle .
\end{aligned}
$$

The integral contains the fourth-order correlation function of the electric fields. The treatment thereof has been considered earlier in the framework of time domain experiments on laser-induced electrostrictive gratings [6,7]. Assuming the pump field $u(t)$ to be a Gaussian random variable, the fourth-order correlation function factorizes [8],

$$
\begin{aligned}
\left\langle u(t) u^{*}\right. & \left.\left(t-\tau_{d}\right) u^{*}\left(t^{\prime}\right) u\left(t^{\prime}-\tau_{d}\right)\right\rangle \\
= & \left\langle u(t) u^{*}\left(t-\tau_{d}\right)\right\rangle\left\langle u\left(t^{\prime}-\tau_{d}\right) u^{*}\left(t^{\prime}\right)\right\rangle \\
& +\left\langle u(t) u^{*}\left(t^{\prime}\right)\right\rangle\left\langle u^{*}\left(t-\tau_{d}\right) u\left(t^{\prime}-\tau_{d}\right)\right\rangle \\
= & \Gamma\left(\tau_{d}\right) \Gamma\left(-\tau_{d}\right)+\Gamma\left(t-t^{\prime}\right) \Gamma\left(t^{\prime}-t\right),
\end{aligned}
$$

in which we assumed stationarity, so that $\left\langle u(t) u^{*}\left(t^{\prime}\right)\right\rangle=$ $\Gamma\left(t-t^{\prime}\right)$. [A third term in Eq. (2) vanishes because it involves an average over optical frequencies.] Assuming Gaussian statistics, the second-order correlation functions are given by $\Gamma(t)=\exp \left(-\imath \omega_{0} t\right) \exp \left(-t^{2} / \tau_{c}^{2}\right)$ [9], with $\omega_{0}$ the carrier frequency and $\tau_{c}$ the field correlation time of the pump laser. Finally, for the pulse envelope, we also assume a Gaussian shape, with pulse duration $\tau_{p}$, that is, $a^{2}(t)=$ $P^{2} \exp \left(-t^{2} / \tau_{p}^{2}\right)$ with $P^{2}=I_{p} / \tau_{p} \sqrt{\pi}$, so that $a^{2}(t)$ is normalized to the pulse energy of the pump laser, $I_{p}$. The integrations in the forcing spectrum can then be worked out analytically, yielding

$$
\begin{aligned}
\left\langle|A(\omega)|^{2}\right\rangle= & I_{p}^{2} \exp \left(-\frac{\tau_{d}^{2}}{2 \tau_{p}^{2}}\right) \times\left[\exp \left(-\frac{\tau_{d}^{2}}{\tau_{c}^{2}}\right)\right. \\
& \left.\times \exp \left(-\frac{1}{2} \omega^{2} \tau_{p}^{2}\right)+\gamma \exp \left(-\frac{1}{2} \gamma^{2} \omega^{2} \tau_{p}^{2}\right)\right],
\end{aligned}
$$

in which $\gamma^{2}=\left(1+2 \tau_{p}^{2} / \tau_{c}^{2}\right)^{-1}$. The overall strength of the spectral forcing is determined by the temporal overlap of the two Gaussian pump pulses. Its spectral structure consists of two components, a coherent and an incoherent one. The frequency bandwidth of the coherent component, $\Delta \nu_{\text {coh }}$, is determined by the pulse duration, and its amplitude vanishes if the delay between the pulses considerably exceeds the coherence time $\left(\tau_{d} \gg \tau_{c}\right)$. The incoherent component is broader by a factor of $\gamma^{-1}>1$, which involves the pulse coherence times and which persists, irrespective of the delay time between the two pulses.

In the case of zero time delay, this pump spectrum takes on a particularly simple form. Since, in the case of a broadband Nd:YAG laser, the pump pulse duration far exceeds its coherence time, $\tau_{p} \gg \tau_{c}$, the incoherent component is both much broader, with a frequency bandwidth $\Delta \nu_{\text {inc }}$ determined by $\tau_{c}$, and much weaker, by a factor of $\tau_{c} / \sqrt{2} \tau_{p} \ll 1$, than the coherent component. Using representative values for broad-band Nd:YAG lasers of $\tau_{p}=$ $5 \mathrm{~ns}$ and $\tau_{c}=25 \mathrm{ps}$, we find (widths expressed as full width at half maximum, FWHM) $\Delta \nu_{\text {coh }} \approx 75 \mathrm{MHz}$, $\Delta \nu_{\mathrm{inc}} \approx 30 \mathrm{GHz}$, and $\tau_{c} / \sqrt{2} \tau_{p}=0.0035$. Evidently, in the case of CRBS experiments using broad-band $\mathrm{Nd}$ : YAG lasers for pumping [1,3,4], only the incoherent component has sufficient bandwidth to drive the full RayleighBrillouin spectrum of the scattering medium, but, under conditions of perfectly matched pump beam path lengthsthat is, zero time delay-this component becomes swamped by the much stronger, but narrow-band coherent contribution. The latter, however, can be suppressed by introducing a delay time between the two pump pulses that is long relative to the coherence time but short relative to the pulse duration. Thus, we conclude that, in spite of its name, all experiments to date on coherent RBS have, in fact, been conducted in the incoherent regime.

Our experimental setup (Fig. 1 [1]) follows the twodimensional backward scattering configuration of Pan et al. [4] and that of Grinstead and Barker [2]. Two broad-band pump laser beams with nominal wave vectors $\mathbf{k}_{1}$ and $\mathbf{k}_{2}$ are focused with $500 \mathrm{~mm}$ focal-length lenses and cross at their foci under an angle of $178^{\circ}$. The counterpropagating beams induce a periodic dipole force field with wave vector $\mathbf{k}=\mathbf{k}_{2}-\mathbf{k}_{1}$ perpendicular to the fringes. This force field gives rise to density gratings. Because of the broad-band nature of the pump beams, the frequency range of the induced gratings spans the whole bandwidth of the pump beams.

The density waves are probed by the Bragg diffraction of a narrow-band laser with wave vector $\mathbf{k}_{p}$ off the induced density gratings. Phase matching requires that the signal beam $\mathbf{k}_{s}$ propagates in the opposite direction to the pump beam $\mathbf{k}_{1}$, and $\mathbf{k}_{2}-\mathbf{k}_{1}=\mathbf{k}=\mathbf{k}_{s}-\mathbf{k}_{p}$. The signal beam maintains the probe beam's characteristics, such as its polarization, but it will be spectrally broadened due to the interaction with the broad superposition of acoustical waves with frequency $\omega_{s}-\omega_{p}=\omega=\omega_{1}-\omega_{2}$.

We scatter off ambient air. The pump laser is a $Q$-switched, frequency-doubled, broad-band, Nd:YAG laser 
(manufactured by Quantel). The narrow-band probe laser is an injection-seeded, frequency-doubled, pulsed Nd:YAG laser with a FWHM of $150 \mathrm{MHz}$ and an $8 \mathrm{~ns}$ pulse duration (specified by the manufacturer, SpectraPhysics). Typical pulse energies are $8 \mathrm{~mJ}$ and $2 \mathrm{~mJ}$ for the pump and probe beams, respectively. As the peak power densities remain much smaller than $10^{15} \mathrm{~W} \mathrm{~m}^{-2}$, our experiment is in the perturbative regime [10].

The probe beam is polarized perpendicularly with respect to the polarization of the pump beams, and the signal beam is separated from the pump beam path using a thin film polarizer. This arrangement avoids interferences between pump and probe beams.

A customized fiber-coupled Fizeau spectrometer (Angstrom Co Ltd, HighFinesse $\mathrm{GmbH}$ ) is used to measure the scattered frequency distribution. The Fizeau spectrometer is based on the same fundamental principles of multiple beam interference as a Fabry-Perot etalon [11]. The spectral resolution of our experiments was measured with the probe laser, and the resulting response $\propto$ $\exp \left(-f^{2} / \sigma_{s}^{2}\right)$ has a Gaussian width $\sigma_{s}=140 \mathrm{MHz}$ $(\mathrm{FWHM}=233 \mathrm{MHz})$, which is a combination of the probe laser linewidth and the resolving power of the Fizeau spectrometer.

In order to induce an adjustable time delay $\tau_{d}$ between the two excitation pulses, a series of plan-parallel glass blocks can be inserted into the path of one of the pump laser beams ( $\mathbf{k}_{2}$ in Fig. 1). The total thickness of the blocks was varied in small steps such as to span a delay of $130 \mathrm{ps}$. The signal intensity is corrected for attenuation of the pump beam by the glass delay line (mainly reflection losses). Reported spectra are averages over 1000 laser shots.

The result of the experiment is shown in Fig. 2. Figure 2(a) displays the recorded spectra as a function of delay time between the arrival of the two laser pulses in their common focus; for clarity, these spectra have been normalized (constant area). These spectra correspond to the product of the forcing spectrum and the RayleighBrillouin response of the scattering medium [4]. Under the conditions of the present experiment, air under ambient conditions, the Rayleigh-Brillouin response is close to Gaussian, as seen in the frontmost spectrum of Fig. 2(a). For large delay times $\left|\tau_{d}\right|$, the induced gratings span a broad spectrum and the true spectrum is registered. Around $\tau_{d}=0$, however, the forcing spectrum is dominated by the sharp coherent peak, and so is the registered spectrum. In order to extract the coherent contribution to the pump spectrum, we subtract an average of the spectra recorded at large delay times from all spectra in Fig. 2(a). The result, $I(f)$, is then fitted to a Gaussian curve, $I(f)=$ $a+b \exp \left(-f^{2} / \sigma^{2}\right)$, as illustrated for one of the spectra close to $\tau_{d}=0$ in Fig. 2(b) (dashed line). We find that $\sigma=$ $180 \mathrm{MHz}(\mathrm{FWHM}=300 \mathrm{MHz})$, essentially independent of $\tau_{d}$. Finally, the height of the central peak (that is, the coherent and incoherent contributions), $I_{m}$, is plotted as a
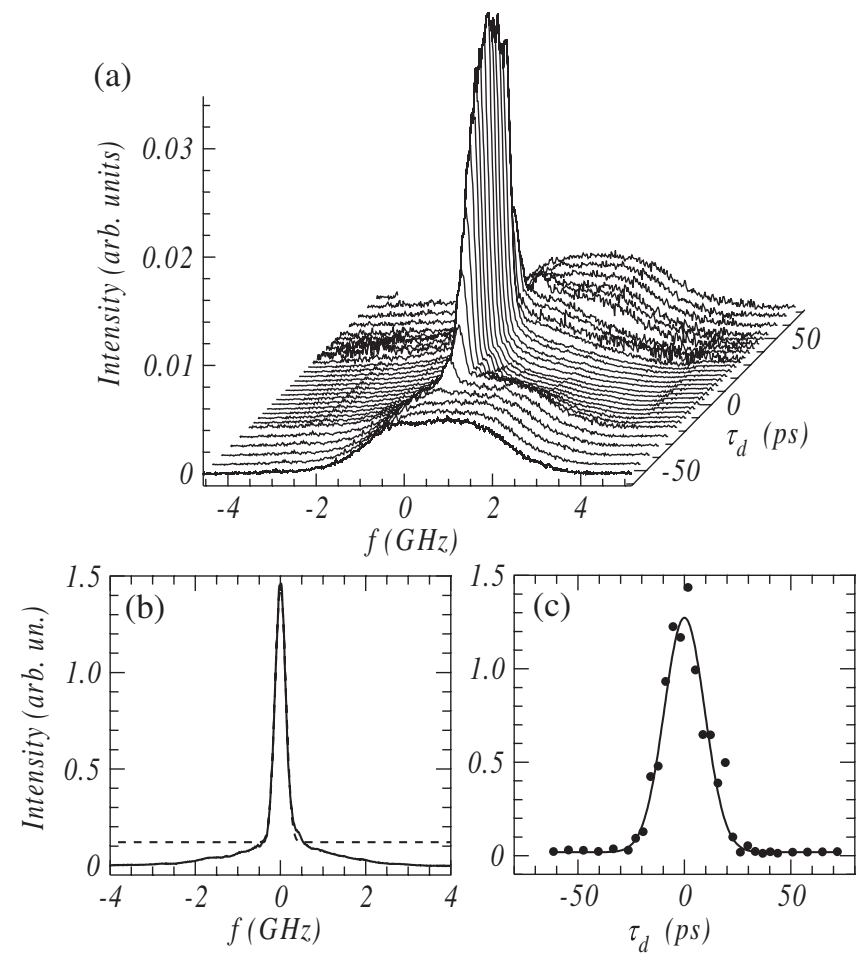

FIG. 2. (a) Coherent Rayleigh-Brillouin spectra $I(f)$ measured as a function of time delay $\tau_{d}$. For clarity, the spectra have been intensity normalized, $\int I(f) d f=1$; this highlights the spectra at large $\left|\tau_{d}\right|$. (b) The solid line indicates the spectrum at $\tau_{d}=0$; the dashed line is the Gaussian fit, $I(f)=b+a \exp \left(-f^{2} / \sigma^{2}\right)$, with $\sigma=182 \mathrm{MHz}$. (c) The dots are peak heights $I_{m}=a+b$, with $a$ and $b$ from fits such as shown in (b), as a function of delay time $\tau_{d}$. The solid line is the Gaussian fit $I_{m}\left(\tau_{d}\right)=$ $\alpha_{m}+\beta_{m} \exp \left(-\tau_{d}^{2} / \tau_{c}^{2}\right)$, with $\tau_{c}=12$ ps and $\beta_{m} / \alpha_{m}=73$.

function of delay time $\tau_{d}$ and again fitted to a Gaussian function, $I_{m}=\alpha_{m}+\beta_{m} \exp \left(-\tau_{d}^{2} / \tau_{c}^{2}\right)$. The result is shown in Fig. 2(c); we find $\tau_{c}=12$ ps and $\beta_{m} / \alpha_{m}=$ $\gamma^{-1}=73$. We take the location of the maximum of this fit to be the factual $\tau_{d}=0$; the time axis of Fig. 2(a) has been adjusted accordingly.

We can compare these results to the Gaussian prediction of the grating spectrum, Eq. (3). First, we realize that, in our experiment, $\tau_{p} \gg\left(\tau_{d}, \tau_{c}\right)$, so that we can approximate $\gamma \approx \tau_{c} / \sqrt{2} \tau_{p}$. Two limiting cases of Eq. (3) then become

$$
\begin{aligned}
& \left\langle\left|A\left(\omega \mid \tau_{d}=0\right)\right|^{2}\right\rangle \propto e^{-\omega^{2} \tau_{p}^{2} / 2}+\frac{\tau_{c}}{\sqrt{2} \tau_{p}} e^{-\omega^{2} \tau_{c}^{2} / 4}, \\
& \left\langle\left|A\left(\tau_{d} \mid \omega=0\right)\right|^{2}\right\rangle \propto e^{-\tau_{d}^{2} / \tau_{c}^{2}}+\frac{\tau_{c}}{\sqrt{2} \tau_{p}} .
\end{aligned}
$$

The spectrum at zero delay is dominated by the first (coherent) term in Eq. (4), a narrow spike with frequency bandwidth $\Delta \nu \propto \tau_{p}^{-1}$, and contains only a small incoherent broad-band contribution $\left(\tau_{c} / \sqrt{2} \tau_{p}\right) \exp \left(-\omega^{2} \tau_{c}^{2} / 4\right)$. Figure 2(b) shows this spectrum, multiplied by the relatively broad Rayleigh-Brillouin response. Our result of 
$300 \mathrm{MHz}$ for the central peak must be considered an upper limit of the actual width, because it is comparable to the estimated bandwidth of the probe laser. It corresponds to a (lower limit of the) pulse duration $\tau_{p}=1.2 \mathrm{~ns}$.

At the central frequency, $\omega=0$, the intensity of the grating spectrum should be described by Eq. (5). Our experimental result is shown in Fig. 2(c), from which we compute (from a Gaussian fit) $\tau_{c}=12 \mathrm{ps}$, corresponding to a bandwidth of $31 \mathrm{GHz}$ (FWHM) of the pump laser. This fit also provides an estimate for the ratio of the Gaussian peak height over the background, $\beta_{m} / \alpha_{m}=73$. According to Eq. (5), this should correspond to the ratio $\gamma^{-1}=\sqrt{2} \tau_{p} / \tau_{c}=140$, in which the numerical value is calculated from the experimentally determined lifetimes.

There is a factor of 2 difference between the estimates of $\gamma^{-1}$, which seems reasonable, given the assumptions made. In deriving Eq. (3), we have assumed a Gaussian correlation function of the optical field and a Gaussian pulse envelope. Also, the width of the coherent spike in the spectra of Fig. 2(a) is determined to a significant extent by the frequency resolution of our experiment (probe laser and Fizeau spectrometer), but this will affect both our estimate of $\tau_{p}$ (width) and that of $\beta_{m}$ (area) in about the same way. We conclude that our description of the forcing spectrum Eq. (3) is adequate and allows us to predict how the coherent component of the forcing spectrum can be exploited.

Figure 3 shows the forcing spectrum for zero time delay, $\tau_{d}=0$, as a function of pump laser pulse duration, as predicted by Eq. (3). The figure plots $\left\langle|A(\omega)|^{2}\right\rangle / I_{P}^{2}$ in a linear gray scale against the (real) frequency and the logarithm of the pump pulse length in units of the pump pulse coherence time, fixed at the value found above, $\tau_{c}=$ 12 ps. As discussed earlier, in the case of long pulse duration $\left(\tau_{p} \rightarrow 100 \tau_{c}\right)$, the spectrum is dominated by the narrow coherent component. If the pulse duration decreases, this coherent component broadens, and simultaneously the incoherent component becomes stronger. These two effects combine to result in a significant spectral broadening.

For the special case of Fourier-limited pulses, we must have temporal overlap within the coherence time, $\tau_{d}<\tau_{c}$. Setting $\tau_{d}=0$ and $\tau_{c}=\tau_{p}$, the forcing spectrum Eq. (3) now is the sum of two broad-band contributions

$$
\left\langle|A(\omega)|^{2}\right\rangle=I_{p}^{2}\left[\exp \left(-\frac{1}{2} \omega^{2} \tau_{c}^{2}\right)+\frac{1}{\sqrt{3}} \exp \left(-\frac{1}{6} \omega^{2} \tau_{c}^{2}\right)\right] .
$$

As compared to the equally broad, but incoherent forcing using nanosecond pump pulses [second term in Eq. (5)], the use of Fourier-limited picosecond pulses provides more than a factor of 200 increase in signal strength [factor $\left(1+3^{-1 / 2}\right) \sqrt{2} \tau_{p} / \tau_{c}$ at $\left.\omega=0\right]$.

Thus, we predict that coherent Rayleigh-Brillouin scattering performed with bandwidth-limited picosecond pump laser pulses will benefit from about 2 orders of

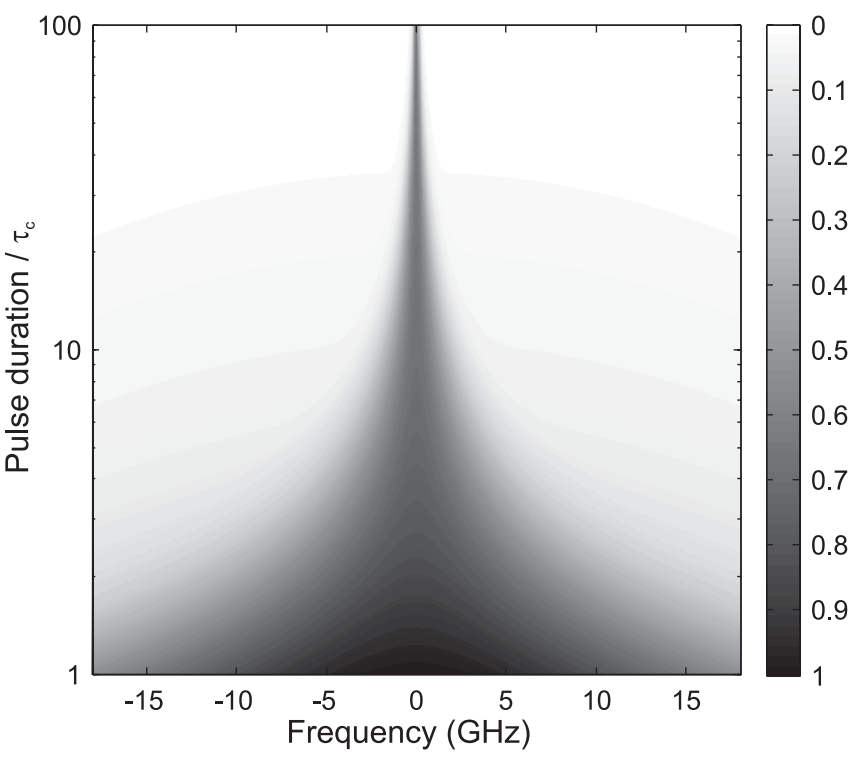

FIG. 3. Normalized amplitude of the forcing spectrum for zero delay as a function of pump laser pulse duration. The amplitude is plotted in a linear gray scale; pump pulse duration in units of the coherence time, $\tau_{c}=12 \mathrm{ps}$, is logarithmic.

magnitude gain in signal strength, as compared to the case of regular nanosecond pulses of the same energy. This should allow spectra to be recorded on the basis of single laser pulses. If we estimate the size of the induced perturbation of the molecular velocity distribution as the momentum gain in half a period of the sound wave or within the pump pulse length, whichever is shorter, these short pulses should still be considered as perturbative.

*Corresponding author.

A.Manteghi@tue.nl

[1] A. S. Meijer, A. S. de Wijn, M. F. E. Peters, N. J. Dam, and W. van de Water, J. Chem. Phys. 133, 164315 (2010).

[2] J. H. Grinstead and P. F. Barker, Phys. Rev. Lett. 85, 1222 (2000).

[3] X. Pan, M. N. Shneider, and R. B. Miles, Phys. Rev. Lett. 89, 183001 (2002).

[4] X. Pan, M. N. Shneider, and R. B. Miles, Phys. Rev. A 69, 033814 (2004).

[5] M. N. Shneider, P. F. Barker, X. Pan, and R. B. Miles, Opt. Commun. 239, 205 (2004).

[6] R. Trebino, E. K. Gustafson, and A. E. Siegman, J. Opt. Soc. Am. B 3, 1295 (1986).

[7] A. Stampanoni-Panariello, B. Hemmerling, and W. Hubschmid, Phys. Rev. A 51, 655 (1995).

[8] J. Goodman, Statistical Optics (Wiley, New York, 1985).

[9] R. Loudon, The Quantum Theory of Light (Oxford University Press, Oxford, 1983), 2nd ed..

[10] H. T. Bookey, M. N. Shneider, and P. F. Barker, Phys. Rev. Lett. 99, 133001 (2007).

[11] J. R. Rogers, J. Opt. Soc. Am. 72, 638 (1982). 